\title{
Utilizing Coffee Ground Waste to Enhance Polyhydroxyalkanoates (PHA) Derived from Soil Bacteria
}

\author{
Eunice Rhee \\ Seoul International School, 15, Seongnam-daero 1518 beon gil, Sujeong-gu, Seongnam-si, Gyeonggi-do, 13113, Republic of Korea; \\ gosyber@suwon.ac.kr
}

\begin{abstract}
Bioplastics are known to have many benefits for the environment, such as slowing down global warming by reducing greenhouse gases and preventing environmental pollution caused by plastic waste. Due to these advantages, it is considered a future-oriented industry, with diverse studies progressing for its widespread use. However, despite its numerous actual and potential uses, the high production costs hinder bioplastic from completely replacing conventional plastics. Therefore, the focus of this research was to develop a method to increase the production efficiency of PHA (Polyhydroxyalkanoates), a natural polymer with high similarity to synthetic plastic, using coffee extract waste. This not only solves the existing environmental problems caused by coffee waste, but also serves as a rich carbon source for bacteria to increase the PHA production rate. Through this experiment, B. Megaterium was successfully selected as our choice of PHA producing soil bacteria. Also, coffee oil was efficiently extracted using a stovetop espresso maker and the inclusion of $3 \%$ coffee oil was found to significantly increase PHA production in B. Megaterium. In conclusion, this research not only provides a solution for this coffee waste problem, but also improves the production of PHA in bacteria and possibly reduces the production costs of bioplastics.

KEYWORDS: Microbiology; Environmental Microbiology; Soil Bacteria; Bioplastic; PHA.
\end{abstract}

\section{- Introduction}

Lack of nutrients in an environment can often lead to competition within bacterial populations. In these conditions, bacteria utilize numerous strategies, one of which is storing nutrients not only for the enhancement of their own survival, but also to deprive its competitors of them. ${ }^{1} \mathrm{PHA}$ (Polyhydroxyalkanoates) is able to fulfill their role as storage units for cells in challenging environmental conditions by storing excess carbon and energy. ${ }^{2}$ Thus, harsh conditions or presence of nutrients may be a determining factor in the frequent occurrence of PHA in bacteria.

PHA, a type of natural polymer, is a prokaryotic storage macromolecule, which is collected as water insoluble granules in bacteria cytoplasm. ${ }^{3}$ PHA is composed of repeating monomers of $\beta$-hydroxyalkanoic acids joined by bonds between the carboxyl group and the hydroxyl group. ${ }^{4} S c l$-PHA consists of monomers of three to five carbon atoms, exhibiting thermoplastic properties. $\mathrm{Mcl}$-PHA consists of monomers of six to 16 carbons and demonstrates latex-like features. Blends of these properties help make up for the unfavorable drawbacks that may be present when produced as bioplastic. Still, PHA has numerous benefits, such as biodegradability, biocompatibility, availability in numerous renewable resources, and the highest similarity to standard chemically synthesized plastics, and this has led to the popularity of its integration in the production of sustainable products. ${ }^{5}$

In order to become an adequate replacement for normal plastics, the production costs need to be minimized, while the productivity is maximized. ${ }^{6}$ When producing biodegradable plastic from soil bacteria, one major problem that greatly increases the costs is the lack of a sufficient carbon source. ${ }^{7}$
This causes the need for more bacteria in the production of bioplastic. ${ }^{8}$

Due to this reason, many researchers have been searching for a prospective, inexpensive substrate that may provide fatty acids, as they are utilized during a $\beta$-oxidation pathway that directly produces acetyl-CoA, a precursor of PHA production. ${ }^{4}$ Plant oils are highly suitable because out of the other inexpensive carbon sources, the theoretical yield of PHA production from plant oils is found to be up to $1.0 \mathrm{~g}$ of PHA per gram of plant oil, which is a much higher quantity than others. ${ }^{9}$

As a substance derived from a plant, coffee oil is composed of high quantities of carbon atoms per weight. ${ }^{10}$ Therefore, it was utilized in the experiment because it had the greatest production of PHA out of the other possible waste or inexpensive oils. ${ }^{11} \mathrm{In}$ the coffee industry, most of the ground coffee wastes are either burned or discharged into the environment. ${ }^{12}$ As the industry grows, the burden of disposal also seems to be exacerbated. The use of coffee oil as a carbon source can therefore be beneficial in multiple ways, as it not only provides a solution for the coffee waste problem, but also improves the production of PHA in bacteria and possibly reduces the production costs of bioplastics.

\section{- Methods}

\section{Agar plate preparation for soil bacteria:}

Nile red staining was used to specify the types of bacteria that would contain PHA. $5 \mathrm{mg}$ per $1 \mathrm{~mL}$ of the solvent, DSMO, was first used to make the Nile red stock solution. ${ }^{13}$ Then, $1.5 \mathrm{~g}$ of agar, $2.5 \mathrm{~g}$ of LB, $50 \mathrm{~mL}$ of water, and $50 \mu \mathrm{L}$ of the Nile Red stock solution were combined and microwaved to make the solution on an agar plate. To ensure that all plates had an equal amount of solution, $9 \mathrm{~mL}$ was poured in each 
of the 5 agar plates. and the elbow movement. A micro servo was positioned on the wrist part and a high torque servo on the elbow part.

\section{Optimization of culture condition:}

In order to determine the most favorable concentration, three different concentrations were tested on separate plates. A soil sample containing about 40-50 g was first collected in a $50 \mathrm{~mL}$ tube from Seoul, Dogok-dong. Then, $0.1 \mathrm{~g}$ of this soil sample was measured and moved to a labeled $15 \mathrm{~mL}$ tube. $10 \mathrm{~mL}$ of deionized water was then added into this tube using a pipet to create a solution with a soil concentration of 1:100. To mix thoroughly, the solution was vortexed for $30 \mathrm{~min}$. Then, $1 \mathrm{~mL}$ of the solution was evenly distributed on an agar plate using a spreader to make a 1:1,000 sample. The remaining solution was used to further dilute the sample to $1: 10,000,1: 50,000$ and 1:100,000 plate samples. After slightly drying them, the agar plates were placed in a $37^{\circ} \mathrm{C}$ incubator for approximately 16 hours.

\section{Coffee oil extraction from coffee ground:}

To prepare the extraction, coffee grounds were dried at $60{ }^{\circ} \mathrm{C}$ for 72 hours. Then, to extract hydrophobic coffee oil, $50 \mathrm{~mL}$ of N-hexane, which is also a hydrophobic solvent, was placed in the water tank of a stovetop espresso maker. The filter basket contained 20 grams of the dried coffee grounds, while the upper chamber was left empty. The maker was placed on a hot plate stirrer at $69^{\circ} \mathrm{C}$ for 10 minutes to prevent overheating the $\mathrm{N}$-hexane, which has a boiling point of $68^{\circ} \mathrm{C}$.

\section{Selection of PHA producing bacteria:}

When the Nile red agar plates were placed under blue light, the bacteria containing PHA turned pink or red. Out of the hundreds of bacterial colonies, five of the most vibrant pink or red colonies were selected, making sure some of these had varying qualities to guarantee different bacterium types. As a negative control, one colony, which was neither pink nor red, was selected to show the difference in PHA producing rates. These 6 selections were then labeled with a marker on the back of the plate.

\section{PHA extraction from soil bacteria:}

To extract the PHA, first, $1 \mathrm{~mL}$ of LB solution was placed in $1.5 \mathrm{~mL}$ tubes. Then, frozen bacteria stocks were thawed to room temperature and seeded into the $1 \mathrm{~mL} \mathrm{LB}$ media, along with $0 \%, 1.5 \%$ and $3 \%$ coffee oil. Once the bacteria were cultured in a $37^{\circ} \mathrm{C}$ shaking incubator for 16 hours, the tubes were centrifuged for 5 minutes at 4,000 g. After 5 minutes, bacteria were collected as a pellet and the remaining solution was carefully removed. Then, $100 \mu \mathrm{L}$ of chloroform was added to resuspend the bacteria. Once the chloroform was removed, $500 \mu \mathrm{L}$ of chloroform was added. Bacteria were then incubated at $60^{\circ} \mathrm{C}$ to completely dissolve the pellet. The samples were further vortexed for $10 \mathrm{~min}$, centrifuged at $13,000 \mathrm{~g}$ for $5 \mathrm{~min}$. and then carefully moved to a new $1.5 \mathrm{~mL}$ tube. 500 $\mu \mathrm{L}$ of chilled methanol was added to the solution to obtain pure PHA by methanol precipitation. When the final solution was dried in the drying oven for $60^{\circ} \mathrm{C}$ overnight, PHA was obtained in a form of white powder.
Identification of bacteria strain with BLAST sequence alignment:

DNA was extracted and 16S ribosomal RNA gene was specifically amplified by polymerase chain reaction (PCR). After the amplified sequence was analyzed by Sanger sequencing, about $700 \mathrm{bp}$ of nucleotide sequence were identified. Then, this DNA sequence was inserted in nucleotide BLAST as a query sequence.

\section{- Results and Discussion}

Optimizing the concentration of bacteria to grow on an agar plate:

The objective of this experiment was to determine the optimal concentration of bacteria when cultured on an agar plate. This was an essential step to be established prior to the rest of the study because if the concentration of bacteria was too high, lack of space would inhibit the growth of different bacterial colonies. On the other hand, if the concentration of bacteria was too low, the chance of the agar plate containing bacteria with PHA would be decreased. Determining the optimal condition would also be essential because the concentrations that are either too high or too low would make it difficult to extract the bacteria after the Nile Red Staining. The soil was diluted in water with concentrations of $1: 1,000$, $1: 10,000,1: 50,000$, and 1:100,000. After the four solutions were spread evenly on agar plates and placed in the incubator for 16 hours, the optimal concentration was determined to be within 1:10,000 and 1:50,000 (Figure 1).
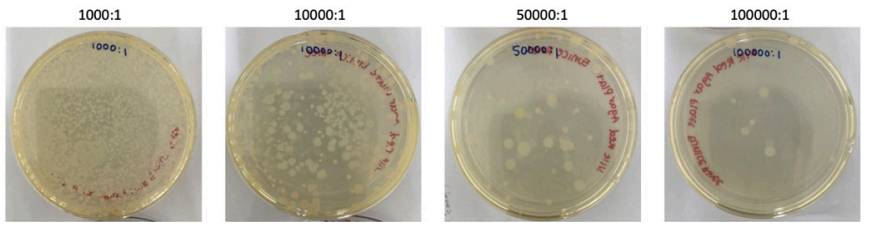

Figure 1: Effect of four different dilution conditions on the number of colonies in an agar plate. The optimal dilution condition was found to be between 1:10,000 and 1:50,000, which shows that more than 40 bacterial colonies were clearly separated from one another.

\section{Selection of PHA producing bacteria:}

When shone under the light, differences in fluorescence were able to be classified. Smaller colonies seemed to have stronger shades of red, while larger groups tended to have lighter shades. Overall, the colonies that exhibited red shades seemed to have a comparably faded outline. (Figure 2)

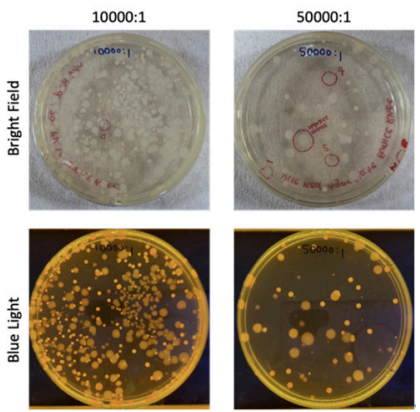

Figure 2: Selection of PHA-producing bacteria by Nile red staining. The figure illustrates the two most suitable concentrations under a blue light. PHA-producing bacteria can be distinguished by red colonies. 


\section{Images of selected bacteria:}

Next, the tip of a pipet was used to select six isolated single colonies: 5 colonies exhibiting red or pink color and 1 negative control colony. These colonies were diluted in $1 \mathrm{~mL}$ of water. $2 \mu \mathrm{L}$ of each selected colony was placed under the microscope to confirm that each sample exhibited only one phenotype, as it would help guarantee that there was no cross contamination. For the experiment, colony \#1 was used due to its highest vibrancy in redness from the Nile red staining. As shown in Figure 3, the bacteria from colony \#1 have long, string-like figures, and when zoomed in further, short sticks-like shapes joined to create its twisting figure.

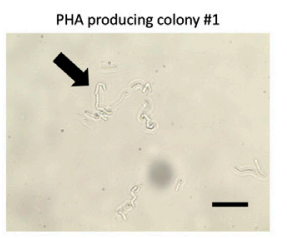

PHA producing colony \#4
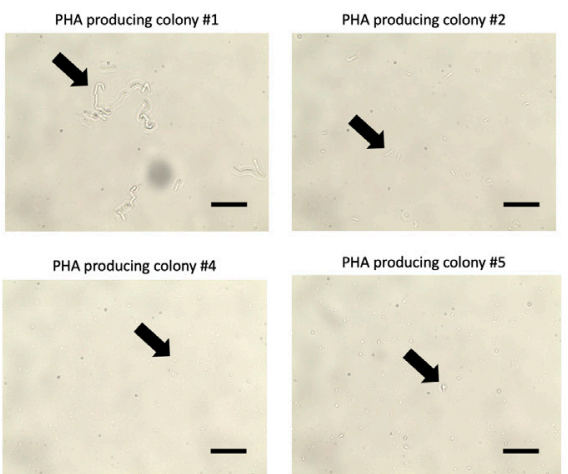

PHA producing colony \#5

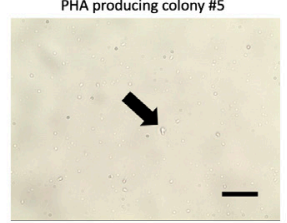

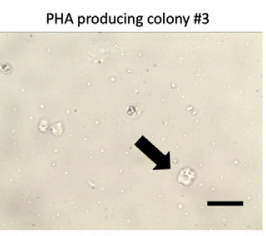

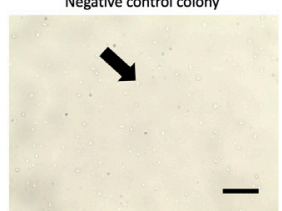

Negative control colony

Figure 3: Microscope images of six selected bacterial colonies exhibiting different phenotypes. Each colony had one corresponding shape, indicating no cross contamination occurred. The arrow indicates the position of bacteria in the image. Scale bar $=10 \mu \mathrm{m}$.

\section{Coffee oil extraction from coffee grounds:}

The stovetop espresso maker that was used for the extraction had three compartments: the water tank, where the N-hexane solvent was placed, the filter basket, where the coffee grounds were placed, and the upper chamber, where the coffee oil would be collected. (Figure 4).

The lid in the upper chamber had a small hole that allowed air to pass through, while the water tank was tightly sealed to generate pressure difference. To summarize how the stovetop espresso maker works, when heated, the hydrophobic solution would vaporize in the water tank, attracting the oil in the coffee grounds as it passes through the filter basket, then it turns back into liquid form as the pressure drops in the upper chamber, creating coffee oil.

Once the coffee oil was extracted by a stovetop espresso maker, N-hexane, which originally had a clear color, now had a brown tint to it, which darkened each time the extraction process was repeated three times. ${ }^{12}$

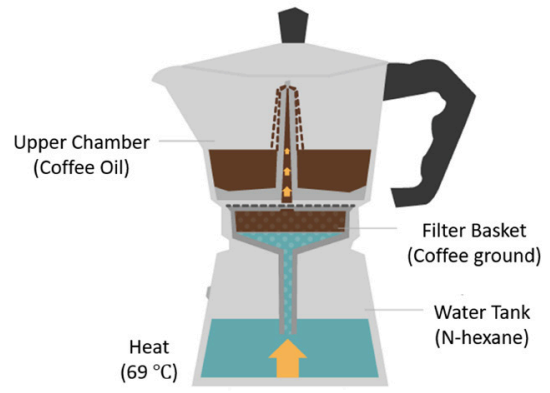

Figure 4: Schematic drawing of stovetop espresso maker as an extractor for coffee oil.

\section{Effect of coffee oil on $\mathrm{PHA}$ production:}

The white powder in each petri dish in Figure 5A shows the extracted PHA from the bacteria. Figure 5A shows how the white powder increases as the percentage of coffee oil supplementation increases. This indicates that the coffee oil enhances PHA production in bacteria.

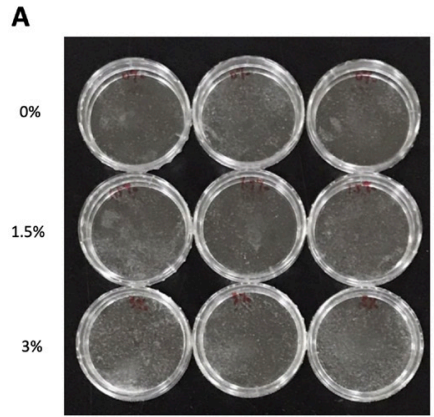

B

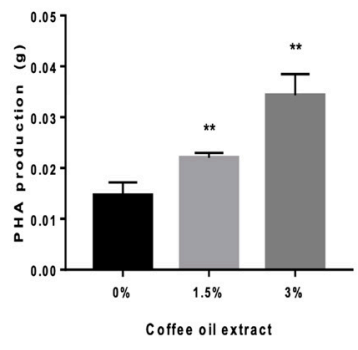

Figure 5: Enhanced PHA production in bacteria by coffee oil supplementation. (A) The image of purified PHA from bacteria. (B) Quantification of PHA production after supplementation with $0 \%, 1.5 \%$, $3 \%$ coffee oil extract. Unpaired t-test was used to identify the statistical significance. $(* *$ indicates p-value less than 0.01$)$.

When the extracted coffee oil was added to the LB solution, it was found that PHA production increased as the percentage of coffee oil increased. When the coffee oil was absent, the PHA production was about $0.015 \mathrm{~g}$, but when $1.5 \%$ of the solution was coffee oil, the PHA production increased to 0.023 g. The PHA production increased even further with $3 \%$ coffee oil to $0.035 \mathrm{~g}$. (Figure 5B) In this experiment, $3 \%$ coffee oil supplementation during bacterial culture showed the highest PHA production.

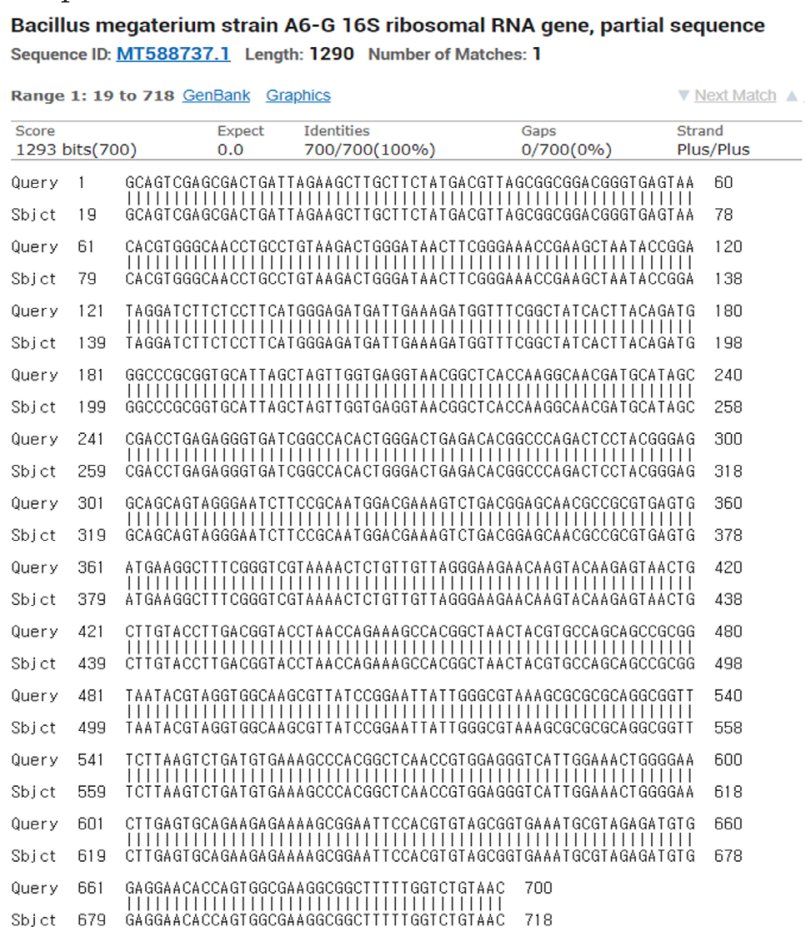

Figure 5: Nucleotide Blast DNA sequence alignment analysis shows that colony \#1 bacteria DNA is identical to B. Megaterium $16 \mathrm{~S}$ ribosomal RNA. Query is an input sequence analyzed from colony \# 1 bacteria. Subject (Sbjct) is a sequence searched within a database. 
BLAST program allows DNA sequences from all organisms including bacteria to be searched. ${ }^{7}$ Since many organisms' genomic sequences are already identified and saved in the genome databases, BLAST is able to provide the best matching sequence from these databases for bacterial identification. When the best matching sequence result was analyzed, the DNA sequence from colony \#1 was found to be identical to B. Megaterium 16S ribosomal RNA (Figure 6). This DNA analysis result indicates that the identity of colony $\# 1$ is $B$. Megaterium.

\section{- Conclusion}

When conducting this experiment, the concentration of bacteria that would be ideal for growth on the agar plate was first determined. While these were left to grow, the coffee ground waste was utilized to extract coffee oil. Once the optimized concentration was specified, the soil bacteria was left to grow in the Nile red agar plate. The reddest colony, which has been identified as B. Megaterium by DNA sequencing, was then selected and prepared for PHA isolation. Three trials of three different concentrations of coffee oil were conducted for reliable results. Through procedures including centrifugation, chloroform washing, and methanol precipitation, pure PHA was obtained. Once this final solution was dried, the PHA was in a form of powder in petri dishes. As the percentage of coffee oil increased, the PHA production also increased, indicating that coffee oil increased the efficiency of the PHA production.

Like most experiments, however, this experiment also had some limitations and compromises. One was the utilization of $\mathrm{N}$-hexane during the coffee oil extraction process. N-hexane is commonly used to extract oils but is also known to have harmful effects on humans. Exposure to such substances may result in damage ranging from central nervous system effects to polyneuropathy in humans. ${ }^{14}$ In future studies, finding a substitute for $\mathrm{N}$-hexane will help enhance the quality of this bioplastic.

To conclude, not only is this method both a highly efficient and cost-effective way to produce PHA, but it also reuses wasted coffee grounds during the production of coffee oil. This issue pertains highly to the authors, as South Korea is ranked third in producing the most plastic waste per person and has coffee consumption rates increasing by $20.3 \% .{ }^{15}$ In the future, this innovative solution may help alleviate the detrimental effects of such problems in South Korea, and possibly, the Earth's environment.

\section{Acknowledgements}

I would like to express my special thanks to my parents and my mentor who gave me the opportunity to work on this wonderful project. Additionally, they helped me in learning more about the scientific procedure and the sustainable production of biodegradable substances.

\section{References}

1. Haruta, S.; Kanno, N., Survivability of Microbes in Natural Environments and Their Ecological Impacts. Microbes Environ 2015, 30 (2), 123-5.

2. Chen, G. Q.; Jiang, X. R., Engineering bacteria for enhanced polyhydroxyalkanoates (PHA) biosynthesis. Synth Syst Biotechnol 2017, 2 (3), 192-197.
3. Somleva, M. N.; Peoples, O. P.; Snell, K. D., PHA bioplastics, biochemicals, and energy from crops. Plant Biotechnol J 2013, 11 (2), 233-52.

4. Sabapathy, P. C.; Devaraj, S.; Meixner, K.; Anburajan, P.; Kathirvel, P.; Ravikumar, Y.; Zabed, H. M.; Qi, X., Recent developments in Polyhydroxyalkanoates (PHAs) production - A review. Bioresour Technol 2020, 306, 123132.

5. Anjum, A.; Zuber, M.; Zia, K. M.; Noreen, A.; Anjum, M. N.; Tabasum, S., Microbial production of polyhydroxyalkanoates (PHAs) and its copolymers: A review of recent advancements. Int J Biol Macromol 2016, 89, 161-74.

6. Zhao, K.; Deng, Y.; Chun Chen, J.; Chen, G. Q., Polyhydroxyalkanoate (PHA) scaffolds with good mechanical properties and biocompatibility. Biomaterials 2003, 24 (6), 1041-5.

7. Amaro, T.; Rosa, D.; Comi, G.; Iacumin, L., Prospects for the Use of Whey for Polyhydroxyalkanoate (PHA) Production. Front Microbiol 2019, 10, 992.

8. Luengo, J. M.; Garcia, B.; Sandoval, A.; Naharro, G.; Olivera, E. R., Bioplastics from microorganisms. Curr Opin Microbiol 2003, 6 (3), 251-60.

9. Budde, C. F.; Riedel, S. L.; Willis, L. B.; Rha, C.; Sinskey, A. J., Production of poly(3-hydroxybutyrate-co-3-hydroxyhexanoate) from plant oil by engineered Ralstonia eutropha strains. $A p p l$ Environ Microbiol 2011, 77 (9), 2847-54.

10.Cruz, M. V.; Paiva, A.; Lisboa, P.; Freitas, F.; Alves, V. D.; Simoes, P.; Barreiros, S.; Reis, M. A., Production of polyhydroxyalkanoates from spent coffee grounds oil obtained by supercritical fluid extraction technology. Bioresour Technol 2014, 157, 360-3.

11.Rathbone, S.; Furrer, P.; Lubben, J.; Zinn, M.;Cartmell, S., Biocompatibility of polyhydroxyalkanoate as a potential material for ligament and tendon scaffold material. J Biomed Mater Res A 2010, 93 (4), 1391-403.

12.Gigliobianco, M. R.; Campisi, B.; Peregrina, D. V.; Censi, R.; Khamitova, G.; Angeloni, S.; Caprioli, G.; Zannotti, M.; Ferraro, S.; Giovannetti, R.; Angeloni, C.; Lupidi, G.; Pruccoli, L.; Tarozzi, A.; Voinovich, D.; Martino, P. D., Optimization of the Extraction from Spent Coffee Grounds Using the Desirability Approach. Antioxidants (Basel) 2020, 9 (5).

13.Spiekermann, P.; Rehm, B. H.; Kalscheuer, R.; Baumeister, D.; Steinbuchel, A., A sensitive, viable-colony staining method using Nile red for direct screening of bacteria that accumulate polyhydroxyalkanoic acids and other lipid storage compounds. Arch Microbiol 1999, 171 (2), 73-80.

14.Ono, Y.; Takeuchi, Y.; Hisanaga, N., A comparative study on the toxicity of $\mathrm{n}$-hexane and its isomers on the peripheral nerve. Int Arch Occup Environ Health 1981, 48 (3), 289-94.

15.Je, Y., Jeong, S., \& Park, T. Coffee consumption patterns in Korean adults: The Korean national health and nutrition examination survey (2001-2011). Asia Pacific Journal of Clinical Nutrition 2014, 23(4), 691-702.

\section{- Author}

Eunice Rhee is currently a student at Seoul International School. She enjoys traveling to new places and trying new foods. She has interests in biology, environmental science, and data science and hopes to major in these fields. 